Bundesgesundheitsbl -

Gesundheitsforsch - Gesundheitsschutz

1999 - 42: 196-205 @ Springer-Verlag 1999

Originalien und Übersichtsarbeiten

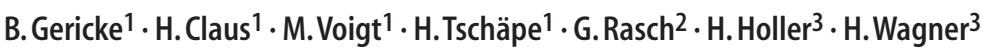

${ }^{1}$ Robert Koch-Institut, Wernigerode

${ }^{2}$ Robert Koch-Institut, Berlin

${ }^{3}$ Labor Dr. Wagner \& Partner, Göttingen

\title{
Die epidemiologische Situation der Salmonellose in Deutschland 1997
}

\section{Vergleich einer Sentinel-Studie mit anderen Datenquellen}

vor allem die Antibiotikaresistenz von S.typhimurium DT 104. Wichtig ist, daß bisher bei Salmonellen nur vereinzelt Resistenzen gegen Ciprofloxacin $(0,2 \%)$ und Cefotaxim $(0,1 \%)$ beobachtet wurden.

Prognostische Einschätzungen der vergangenen Jahre, daß der führende Epidemiestamm S. enteritidis PT 4 rückläufig ist und durch einen neuen aufkommenden Epidemiestamm S.typhimurium DT 104 ersetzt wird, haben sich 1997 nicht erfüllt.

Deutschlands vorliegenden humanen Salmonella-Isolaten sowie stellvertretend für das gesamte Bundesgebiet mit den 29326 nach BSeuchG aus den neuen Bundesländern und Berlin gemeldeten Salmonellen verglichen.

In allen drei Studien wurden zwischen Juli und September etwa 40\% der Salmonellosen beobachtet. Entsprechend lagen in diesem Zeitraum die monatlichen Inzidenzraten zwischen etwa 20 und 30\%. Etwa ein Viertel der Salmonellosen entfiel auf das Säuglings-, Kleinkind- und Vorschulalter, die knappe Hälfte auf die Altersklasse bis 14 Jahre.S. enteritidis und S.typhimurium waren die mit Abstand wichtigsten Serovare.

Bei S. enteritidis war das Resistenzniveau gegen fast alle getesteten Antibiotika mit Resistenzanteilen zwischen 0 bis 2,6\% sehr niedrig. Multiresistente S. enteritidis-Stämme waren mit Anteilen von 1,3\% bzw. 2,8\% zu vernachlässigen. Im Gegensatz dazu zeigte S.typhimurium eine beachtliche Resistenz gegen Ampicillin (A), Chloramphenicol (C), Streptomycin (S), Sulfonamide (Su), Tetracycline $(T)$, besondere Bedeutung hat dabei nfektionen durch Enteritis-Salmonellen (Bakterien der Gattung Salmonella, Spezies und Subspezies S.enterica mit Ausnahme der Serovare S. typhi und S. paratyphi) sind in der Bundesrepublik Deutschland nach wie vor die häufigste erfaßte Ursache von Durchfallerkrankungen. Unsere Kenntnisse zur epidemiologischen Situation der Salmonellose basieren vor allem auf den nach dem Bundes-Seuchengesetz (BSeuchG) vorgeschriebenen Meldungen. Die Zuverlässigkeit der Erfassung auf dem Meldeweg sollte deshalb in gewissen Abständen durch den Vergleich mit anderen Erfassungsmethoden überprüft werden. Hierfür eignen sich z.B. die Auswertungen der Untersuchung von SalmonellaStämmen, die dem Nationalen Referenzzentrum (NRZ) für Salmonellen und andere Enteritiserreger aus allen Regionen
Deutschlands zur epidemiologischen Subdifferenzierung (Typisierung) zugesandt werden, oder die bisher in Deutschland nur relativ selten eingesetzten Sentinel-Studien, die zusätzliche, über die Meldung hinaus vertiefte Informationen liefert.

Ziel solcher Sentinel-Studien ist eine infektionsepidemiologische Untersuchung in Form der aktiven, prospektiven epidemiologischen Überwachung (Surveillance) mit einem ausgewählten Beobachtungslabor (Sentinel) im Rahmen einer vereinbarten ärztlichen $\mathrm{Zu}$ sammenarbeit zur kontinuierlichen Einschätzung der Salmonellose-Inzidenz in einem umschriebenen Gebiet mit einer bestimmten Bevölkerung. Die epidemiologische Überwachung der Salmonellose in Deutschland ist sowohl als Basis für Prävention und Bekämpfung wie auch als Modell für die durch Lebensmittel übertragenen Infektionen wichtig. Darüber hinaus ermöglicht sowohl die Sentinel-Studie als auch die Analyse der Typisierergebnisse der dem NRZ zugesandten Salmonella-Stämme eine eingehende Einschätzung bezüglich der klonalen Ausbreitung von SalmonellaStämmen. Die Sentinel-Studie ist zusätz-

\section{Dr. B. Gericke}

NRZ für Salmonellen und andere Enteritiserreger, Robert Koch-Institut, Bereich Wernigerode, Burgstraße 37,D-38855 Wernigerode 
B. Gericke · H.Claus · M. Voigt · H. Tschäpe · G. Rasch $\cdot$ H. Holler $\cdot$ H. Wagner

The epidemiological situation of salmonellosis in Germany 1997. Comparison of a sentinel-study with other date sources

\section{Summary}

To assess the epidemiological situation of salmonellosis in Germany a total of 1608 strains of salmonella sampled in a sentinelstudy were isolated and typed. The results on occurrence, distribution, types and antbiotic resistance of salmonella were compared with both the strains forwarded to the $\mathrm{Na}$ tional Reference Center (NRC) for Salmonellosis for typing from all regions of Germany and the strains notified according to the duty of notification in the five new Länder (federal countries) and Berlin.

In all three studies about $40 \%$ of salmonellosis were observed between July and September. Approximately a quarter of salmonellosis fell into the age group 0 to 5 years, around half of salmonellosis occurred in the age groups 0 to 14 years. The incidence rates in the sentinel-study and of the notified salmonellosis were highly corresponding with rates of 160.8 and 165.4 , respectively. S. enteritidis and $S$.typhimurium were the most important serovars.

Among the S. enteritidis strains the rates of resistance to the antimicrobial agents used were just $0-2.6 \%$. Also the rate of multiresistant strains of $S$. enteritidis was very low, $1.3 \%$ and $2.8 \%$, respectively. By contrast among the $S$.typhimurium strains high rates of resistance to ampicillin $(A)$, chloramphenicol (C), streptomycin (S), sulfonamide (Su), tetracycline $(T)$ were found. The drug resistance of S.typhimurium DT 104 had a special significance (R-type ACSSuT). About $90 \%$ of the strains tested showed this pattern of drug resistance. Among the other salmonella only the resistance to tetracycline (20\%) and to sulfonamide $(80 \%)$ played a role. The fact is important that up to now among all salmonella resistance to ciprofloxacin (0.2\%) and cefotaxim ( $0.1 \%$ ) was observed rarely. The dominant epidemic strain S. enteritidis PT 4 was not declining and it was not replaced by the emerging epidemic strains S. typhimurium DT 194 so far. lich zur Erfassung von Inzidenzsteigerungen und Ausbrüchen geeignet. In der vorliegenden Arbeit werden die Ergebnisse einer Sentinel-Studie mit den Typisierergebnissen des NRZ und den Meldungen nach dem BSeuchG verglichen.

\section{Material und Methoden}

\section{Sentinel-Studie}

Das Einzugsgebiet des Sentinel-Labors umfaßte Teile des südöstlichen Niedersachsens, des nördlichen Thüringens und des südwestlichen Sachsen-Anhalts mit einer Bevölkerung von etwa einer Million Einwohner. Zur detaillierten Analyse der Salmonellose wurden alle in diesem Labor vom 1.1. bis 31.12.1997 isolierten Salmonellen (1608) dem NRZ übermittelt und einer weiteren Bestimmung und Typisierung zugeführt.

\section{Typisierungen im NRZ}

Zum Vergleich mit den Ergebnissen der Sentinel-Studie wurden alle humanen Salmonella-Stämme (4321) herangezogen, die im gleichen Zeitraum dem NRZ aus allen Regionen Deutschlands zur Typisierung geschickt wurden.

\section{Mikrobiologische Differenzierung, Resistenztestung und Lysotypie}

Die Isolierung, Differenzierung und Identifizierung der Salmonellen erfolgte ebenso wie deren serologische Bestimmung nach den üblichen Standards [1] sowie zusätzlichen Kriterien [2]. Zur Resistenztestung wurde ein modifizierter Mikrobouillonverdünnungstest eingesetzt [3]. Getestet wurden folgende 17 Chemotherapeutika: Ampicillin (AMP), Mezlocillin (MEZ), Mezlocillin/Sulbac$\operatorname{tam}$ (MSU), Cefotiam (CTM), Cefotaxim (CTX), Ceftazidim (CAZ), Cefoxitin (COX), Streptomycin (STR), Gentamicin (GEN), Kanamycin (KAN), Amikazin (AMK), Chloramphenicol (CMP), Oxytetracyclin (OTE), Ciprofloxacin (CIP), Sulfamerazin (SMZ), Trimethoprim/Sulfamerazin (SXT), Nourseothricin (NOT).

Zur Feindifferenzierung diente die Lysotypie. Die Lysotypie der S. typhimurium-Stämme erfolgte mit dem erwei- terten Typisiersystem nach Anderson $[4,5]$. Während international die Phagentypisierung von $S$. enteritidis ausschließlich mit dem System von Ward et al. durchgeführt wird [6], typisierten wir zusätzlich mit dem Lysotypiesystem nach Laszlo et al. [7]. Die beiden Lysotypiebefunde werden getrennt durch einen Schrägstrich angegeben, z.B. LT 4/6 bedeutet LT 4 nach Ward und LT 6 nach Laszlo.

Die Lysotypie von Salmonellen stellt gegenwärtig die einzige durch molekularbiologische Methoden (Genotypie, Elektrotypie) abgesicherte, aber durch diese nicht ersetzbare îSchnellmethode" zur epidemiologischen Aufklärung von Infektketten dar [12].

\section{Meldungen nach BSeuchG}

Da bisher nur in den fünf neuen Bundesländern Brandenburg, MecklenburgVorpommern, Sachsen, Sachsen-Anhalt und Thüringen sowie im Land Berlin neben der Anzahl der isolierten Salmonellen auch die nachgewiesenen Serovare gemeldet werden, dienten ausschließlich die Meldungen des gleichen Zeitraumes aus diesen Bundesländern (29 326) stellvertretend für das gesamte Bundesgebiet zur epidemiologischen Einschätzung der Salmonellose nach BSeuchG.

\section{Ergebnisse}

\section{Monatliche Verteilung und Inzidenz der Salmonellose}

Die monatliche Verteilung der Salmonellose im Vergleich der Sentinel-Studie mit den Typisierungen des NRZ und den Meldungen gemäß BSeuchG ist in Abb. 1 dargestellt. Alle drei Datenquellen weisen einen deutlichen Gipfel in den Sommermonaten und im Frühherbst auf und lassen insgesamt einen fast übereinstimmenden Verlauf erkennen. Abb. 2 zeigt die monatliche Inzidenz der Salmonellose.

Da die Einsendungen an das NRZ auf keine definierte Bevölkerung bezogen werden können, war nur der Vergleich von Sentinel-Studie und Meldungen nach BSeuchG sinnvoll. Auch die aus 

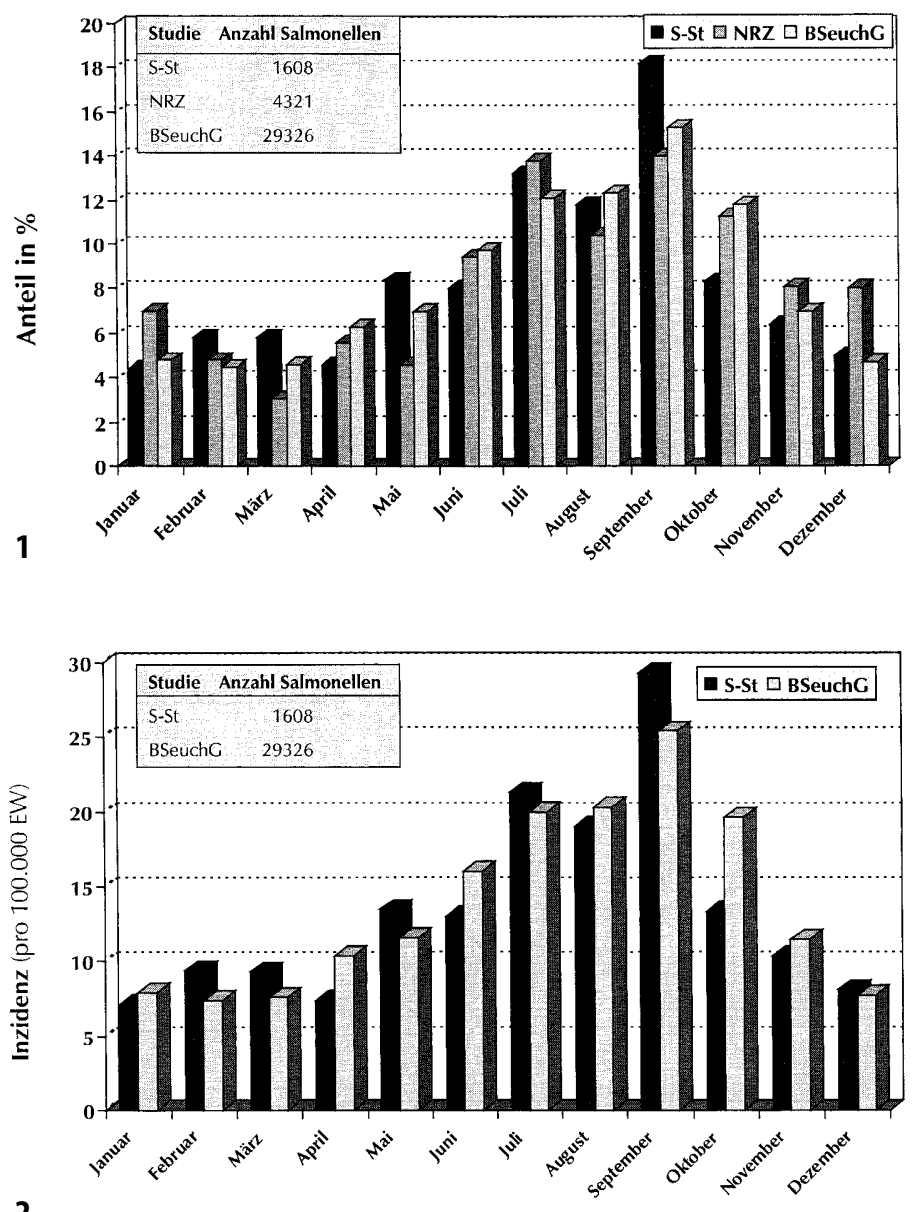

2

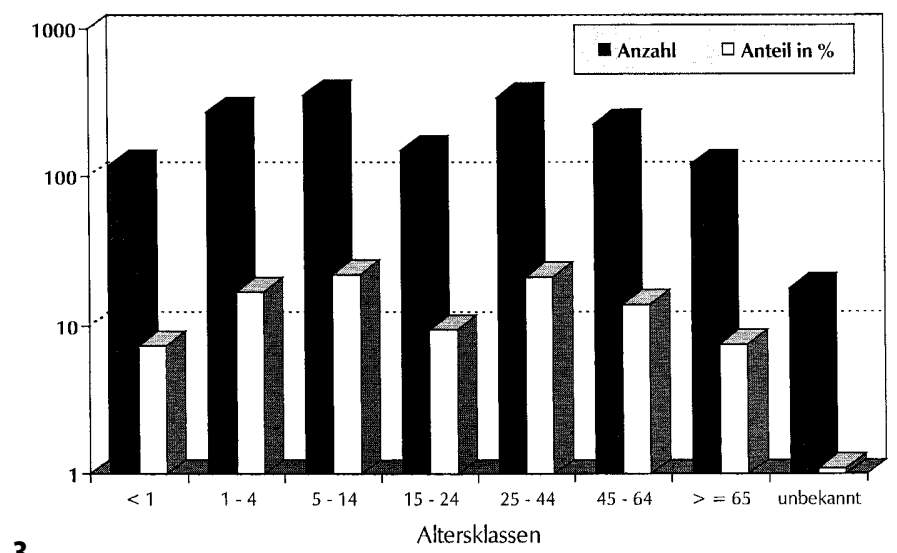

Abb. $1 \Delta$ Monatliche Verteilung der Salmonellose. Vergleich Sentinel-Studie (S-St) 1997, Typisierung humaner Isolate im NRZ 1997 und Meldungen BSeuchG 1997 Abb. 2 Monatliche Inzidenz der Salmonellose. Vergleich Sentinel-Studie (S-St) 1997 und Meldungen BSeuch 1997

Abb. 3 Altersverteilung und altersspezifische Tendenz der Salmonellose Sentinel Studie 1997 der Sentinel-Studie und aus den Meldungen berechneten monatlichen Inzidenzen stimmen mit den Ergebnissen der monatlichen Verteilung der Salmonellose sehr gut überein. In allen drei Studien wurden übereinstimmend zwischen Juli und September etwa 40\% der Salmonellosen beobachtet. Entsprechend lagen in diesem Zeitraum die monatlichen Inzidenzraten zwischen etwa 20 und 30/100 ooo Einwohner.

\section{Altersspezifische Morbidität}

In Abb. 3 sind die Ergebnisse der Sentinel-Studie zur Altersverteilung der Salmonellose zusammengestellt. Die Einteilung der Altersklassen erfolgte entsprechend der inzwischen üblichen Gruppierung [8]. Der Vergleich dieser Daten mit den zur Typisierung an das NRZ eingesandten humanen Salmonella-Stämmen verbot sich aus den oben erläuterten Gründen. Ein Vergleich mit den Meldungen nach BSeuchG wurde nicht durchgeführt, da für die SentinelStudie keine altersspezifische Inzidenzrate berechnet werden konnte. Etwa ein Viertel der Salmonellosen entfielen auf das Säuglings-, Kleinkind- und Vorschulalter, die knappe Hälfte auf die Altersklassen bis 14 Jahre.

\section{Vorkommen und Häufigkeit der Salmonella-Serovare}

Im Verlauf der Sentinel-Studie wurden 40 verschiedene Serovare bestimmt, während bei den Typisierungen im NRZ eine Gesamtzahl von 105 und nach den Meldungen gemäß BSeuchG von 141 Serovaren ermittelt wurde. Im Gegensatz dazu ergab die Zahl der häufigsten Serovare $(n \geq 0,2 \%)$ eine größere Übereinstimmung: für die Sentinel-Studie 19, die Typisierung humaner Isolate im NRZ 34 und die Meldungen nach BSeuchG 18 Serovare.

„Salmonellosen weisen einen Häufigkeitsgipfel in den Sommermonaten sowie im Frühherbst auf und betreffen oft Kinder und Säuglinge." 


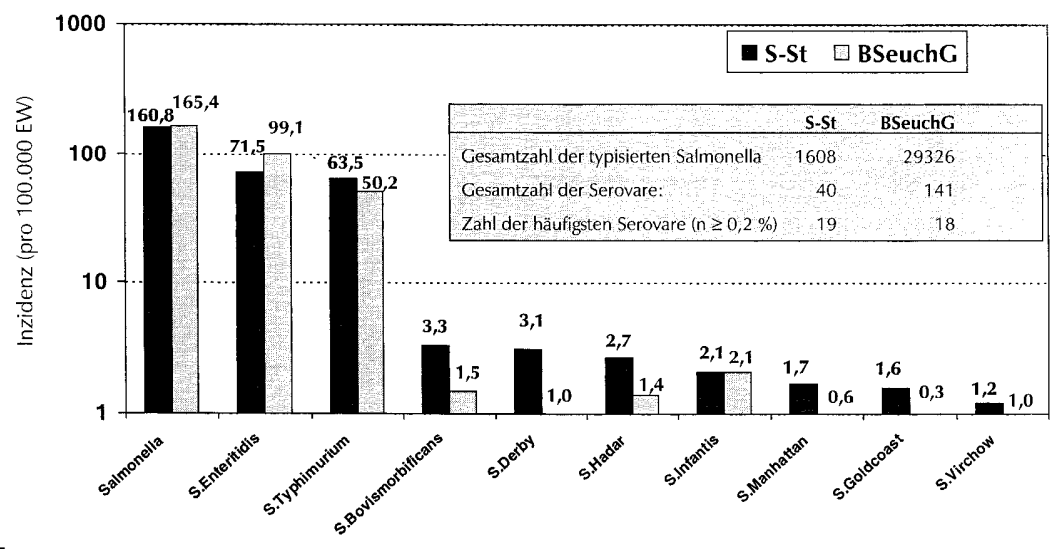

4

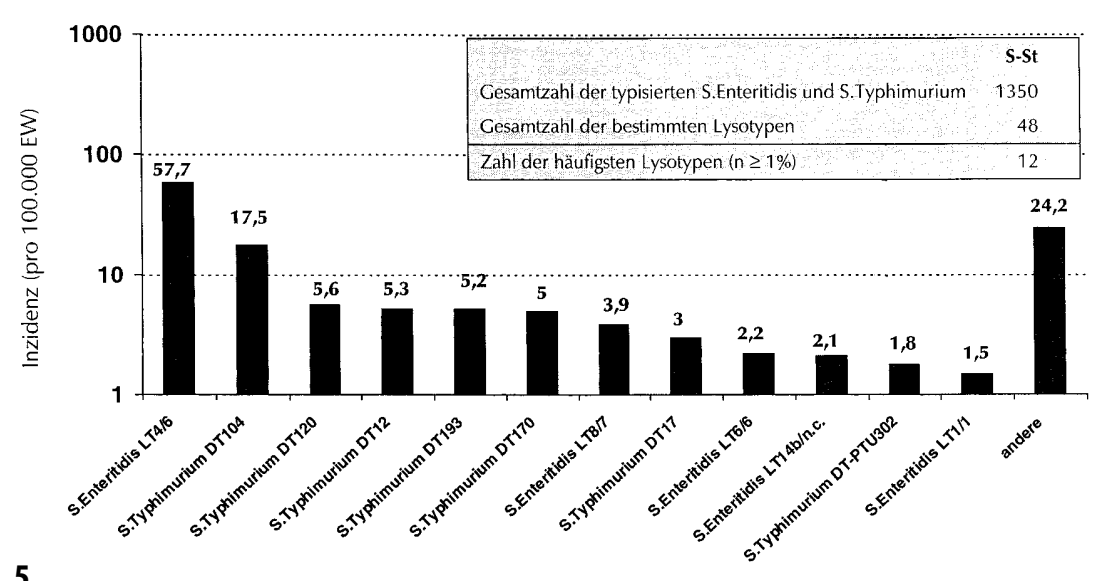

Abb. 4, $5 \Delta$ Inzidenz der häufigsten Lysotypen der Serovare S. enteritidis und S. typhimurium. Sentinel-Studie (S-St) 1997

Die häufigsten Serovare mit der höchsten Inzidenz nach den Ergebnissen der Sentinel-Studie und den Meldungen nach BSeuchG sind der Abb. 4 zu entnehmen. Beide Studien ergaben mit 160,8 und 165,4 eine sehr hohe Übereinstimmung der Inzidenzraten für alle Salmonella-Serovare. Übereinstimmend waren auch S. enteritidis und S. typhimurium die mit Abstand wichtigsten Serovare. Die Inzidenzraten der nächsthäufigen Serovare sind zwar unterschiedlich, jedoch ähnlich.

Für die dominierenden Serovare $S$. enteritidis und S. typhimurium ist aufgrund ihrer besonderen Bedeutung die monatliche Verteilung in Gegenüberstellung von Sentinel-Studie, Typisierung humaner Isolate im NRZ und Meldung nach BSeuchG in Tabelle 1 detailliert aufgeführt. Wie der Aufstellung zu entnehmen ist, sind synchron in allen drei Da- tenquellen beträchtliche jahreszeitliche Unterschiede erkennbar. So war von Januar bis April sowie im November und Dezember übereinstimmend S. typhimurium häufiger nachweisbar als S. enteritidis, während von Juni bis Oktober die Rangfolge der Nachweise umgekehrt war.

\section{Vorkommen und Häufigkeit der Lysotypen von S. enteritidis und S.typhimurium}

Da die Lysotypen (LT) von S. enteritidis und S. typhimurium nach BSeuchG nicht gemeldet werden, war der Vergleich der Ergebnisse der Sentinel-Studie nur mit den Daten der Typisierungen des NRZ möglich. Einzelheiten sind in den Tabellen 2 und 3 detailliert dargestellt.

Tabelle 2 zeigt, daß 80\% der S. enteritidis-Stämme auf LT 4/6 entfielen, was einer Inzidenzrate von 57,7 entsprach.
Bezogen auf alle Salmonellen betrug der Anteil von S. enteritidis LT 4/6 in der Sentinel-Studie 35,9\%, bei Auswertung der Typisierung humaner Isolate im NRZ 30,2\%. Die nächsthäufigen Lysotypen LT 8/7, LT 6/6, LT 14b/n.c., LT 1/1 und LT 21/1 folgten mit großem Abstand bei einem Anteil zwischen 5,5\% und 1,5\%. Alle weiteren S. enteritidis-Lysotypen hatten einen Anteil unter $1 \%$.

Die Zusammenstellung der Lysotypen von S. typhimurium wies keine ähnliche Dominanz eines einzelnen Lysotyps aus (Tabelle 3). Auf den häufigsten Lysotyp S. typhimurium DT104 entfielen 31,5\% (S-St) bzw. 38,1\% (NRZ) der geprüften Stämme, entsprechend einer Inzidenzrate von 20. Bezogen auf alle Salmonellen gehörten zum Lysotyp DT104 in der Sentinel-Studie $12,4 \%$, bei Auswertung der Typisierung humaner Isolate im NRZ 15,8\% der typisierten Stämme. In der Sentinel-Studie hatten die entsprechend der Häufigkeit folgenden Lysotypen DT120, DT12, DT193 und DT17o Anteile zwischen 9,6\% und 8,2\%. Der im Gegensatz zu den Typisierungen des NRZ sehr hohe Anteil des Lysotyps DT186 von 10,7\% war durch einen größeren Ausbruch bedingt.

In der Abb. 5 sind die Inzidenzraten der häufigsten Lysotypen der Serovare $S$. enteritidis und S. typhimurium nach den Ergebnissen der Sentinel-Studie dargestellt. Dabei wurden die im folgenden beschriebenen, durch Ausbrüche bedingten Inzidenzsteigerungen der S. typhimurium-Lysotypen DT104 und DT170 bei der Berechnung berücksichtigt.

\section{Erfassung von Inzidenzsteigerungen und Ausbrüchen}

Im Verlauf der Sentinel-Studie beobachteten wir bei mehreren Salmonella-Serovaren und S. typhimurium-Lysotypen plötzliche Inzidenzsteigerungen. Im einzelnen betraf dies die Serovare S. bovismorbificans, S. derby und S. goldcoast sowie die $S$. typhimurium-Lysotypen DT104, DT170 und DT186.

Die Inzidenzsteigerungen der Serovare wurden im März bis Juni (18 S. bovismorbificans-Stämme), Mai bis Juli (22 S. derby-Stämme) und Juli (15 S. goldcoast-Stämme) registriert. Während des 
gesamten Untersuchungszeitraumes von Januar bis Dezember 1997 wurden insgesamt 33 S. bovismorbificans-, 31 S. derby- und 16 S. goldcoast-Stämme nachgewiesen. Alle Stämme wurden bei regional gestreuten, sporadischen Einzelerkrankungen im nördlichen Thüringen bei zuvor unauffälliger Inzidenz und Prävalenz isoliert. In keinem Fall konnte trotz guter Zusammenarbeit mit den zuständigen Gesundheitsämtern die Infektionsquelle eruiert werden.

Die Inzidenzsteigerungen der S. $t y$ phimurium-Lysotypen konnten im Februar bis März (56 DT186-Stämme), Juni (12 DT170-Stämme) und Juli (26 DT104Stämme) beobachtet werden (Tabelle 3). Im Untersuchungszeitraum der Sentinel-Studie wurden insgesamt 68 DT186Stämme, 61 DT170-Stämme und 200 DT104-Stämme typisiert. Auch für die Inzidenzsteigerung von S. typhimurium DT17o in einigen Orten im Südosten Niedersachsens war die Infektionsquelle nicht nachweisbar. Vermutlich handelte es sich um Hackepeter. Dagegen konnten die beiden durch S. typhimurium DT104 im Südwesten von SachsenAnhalt und DT186 im nördlichen Thüringen bedingten Inzidenzsteigerungen in Zusammenarbeit mit den zuständigen Gesundheitsämtern epidemiologisch geklärt werden. Beide Fälle erwiesen sich als regional weit gestreute Ausbrüche durch den Verzehr von Hackepeter. Über den Ausbruch durch S. typhimurium DT186 haben wir bereits an anderer Stelle ausführlich berichtet [9]. Dabei handelte es sich um einen Ausbruch in zwei benachbarten Landkreisen Thüringens, der ohne diese SentinelStudie unerkannt geblieben wäre.

\section{Antibiotikaresistenz der Salmonellen}

Im Rahmen unserer Sentinel-Studie wurden von allen isolierten 1608 Salmonellen die Antibiotikaresistenz bestimmt und mit den Resistenzbestimmungen der 4321 humanen Salmonella-Stämme verglichen, die dem NRZ zur Typisierung aus allen Regionen Deutschlands zugeschickt wurden. Dabei wurde zum einen eine Auswertung für alle Salmonellen vorgenommen und diese zum anderen den Resistenzwerten von S. enteri- tidis, S. typhimurium, S. typhi und der übrigen Salmonellen gegenübergestellt. Über das Ergebnis dieser Untersuchungen informieren umfassend die Tabellen 4 und 5, wobei aus Tabelle 4 die Resistenz gegen die einzelnen Antibiotika, aus Tabelle 5 der Anteil der sensiblen sowie einfach- und mehrfachresistenten Stämme ersichtlich ist.

Während bei S. enteritidis die Antibiotikaresistenz in beiden Studien sehr nahe beieinander lag, stimmten die übrigen Serovare in ihrer Antibiotikaresistenz weniger stark überein, wobei in der Sentinel-Studie kein S. typhi-Stamm iso- liert wurde. Bei S. enteritidis war insgesamt das Resistenzniveau gegen fast alle getesteten Antibiotika mit Resistenzanteilen zwischen o\% und 2,6\% sehr niedrig. Lediglich für Sulfonamide ergab sich mit über 70\% ein hoher Anteil resistenter Stämme. Multiresistente S. enteritidis-Stämme waren mit Anteilen von 1,3\% bzw. 2,8\% zu vernachlässigen.

Im Gegensatz dazu zeigte S. typhimurium eine beachtliche Resistenz gegen Ampicillin (32,3\%/48,2\%), Tetracycline $(43,5 \% / 59,9 \%)$, Chloramphenicol $(29,1 \% / 38,6 \%)$, Sulfonamid (74,2\%/ $77,4 \%)$ sowie Streptomycin (41,7\%/

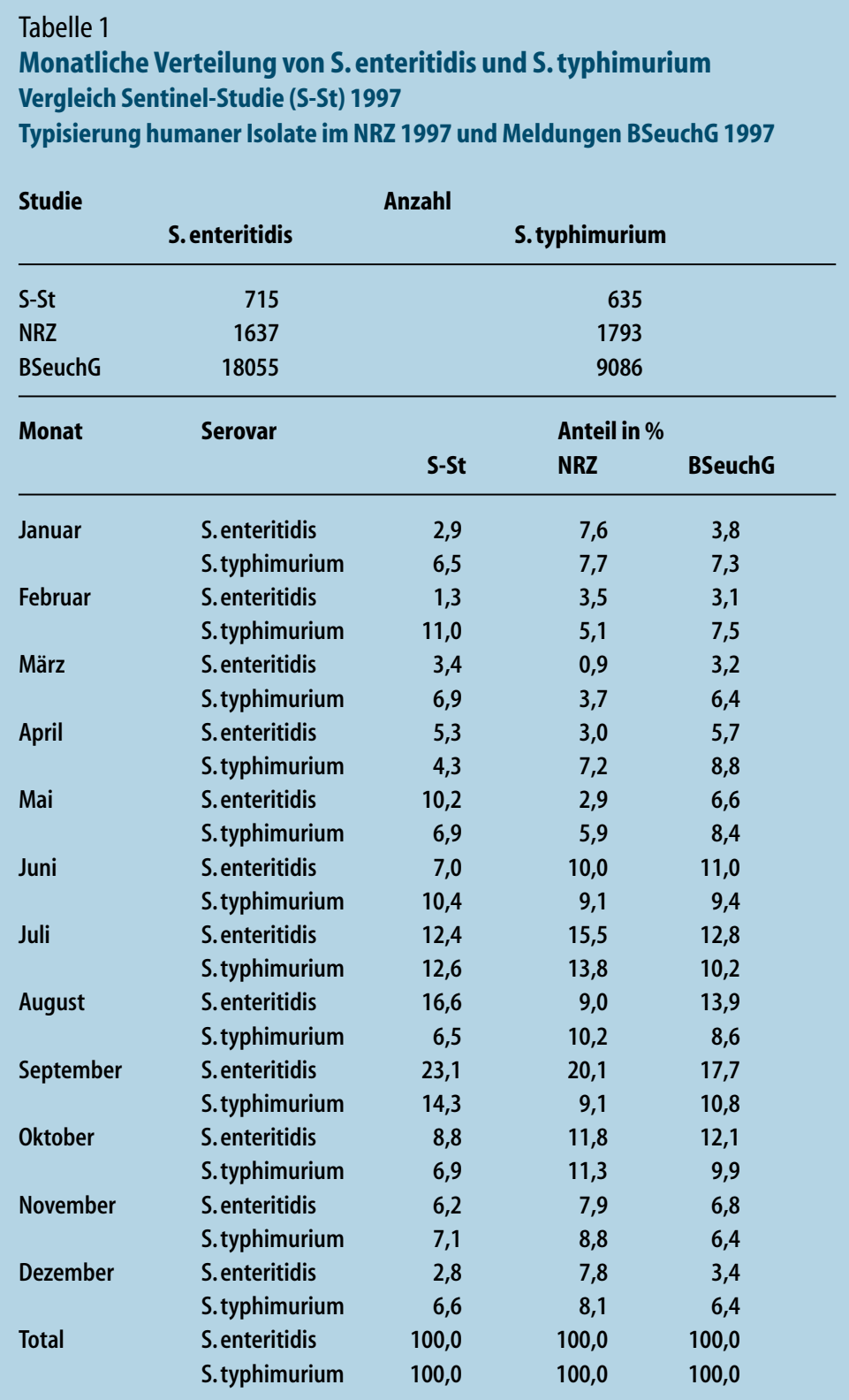


Tabelle 2

Vorkommen und Häufigkeit der Lysotypen von S. enteritidis

Vergleich der Sentinel-Studie (S-St) und Typisierung humaner Isolate im NRZ 1997

\begin{tabular}{lrr} 
& S-St & NRZ \\
\hline Gesamtzahl der typisierten S. enteritidis & 715 & 1637 \\
Gesamtzahl der Lysotypen & 18 & 30 \\
Zahl der häufigsten Lysotypen ( $\mathrm{n} \geq 0,2 \%)$ & 14 & 14 \\
\hline
\end{tabular}

\begin{tabular}{llll} 
Lysotyp & \multicolumn{2}{c}{ Anteil in \% } & Inzidenz* (pro $100000 \mathrm{EW})$ \\
& S-St & NRZ & \\
\hline
\end{tabular}

\begin{tabular}{lrrr}
\hline $4 / 6$ & 80,7 & 79,8 & 57,7 \\
$8 / 7$ & 5,5 & 4,2 & 3,9 \\
$6 / 6$ & 3,1 & 3,5 & 2,2 \\
14 b/n.c. & 2,9 & 2,7 & 2,1 \\
$1 / 1$ & 2,1 & 3,2 & 1,5 \\
$21 / 1$ & 1,5 & 1,8 & 1,1 \\
1 b/1 & 0,8 & 0,1 & 0,6 \\
$36 / 6$ & 0,6 & 0,5 & 0,4 \\
$6 a / 7 a$ & 0,6 & 0,4 & 0,4 \\
$25 / 17$ & 0,4 & 0,3 & 0,3 \\
$7 /$ n.c. & 0,4 & 0,4 & 0,3 \\
n.c./6 & 0,3 & 0,4 & 0,2 \\
n.c./7 & 0,3 & 0,0 & 0,2 \\
5a/7a & 0,3 & 0,4 & 0,2 \\
übrige & 0,6 & 2,1 & 0,4 \\
Total & 100,0 & 100,0 & 71,5 \\
\hline
\end{tabular}

*aus den Daten der Sentinel-Studie berechnet

$57,1 \%)$ und einen hohen Anteil multiresistenter Stämme $(36,5 \% / 54,6 \%)$. In diesem Zusammenhang muß unbedingt zwischen S. typhimurium DT104 und S. typhimurium non DT104 differenziert werden, da sich der Lysotyp DT104 in seinem Resistenzverhalten deutlich von anderen S. typhimurium-Lysotypen unterscheidet.

Besondere Bedeutung hat die Antibiotikaresistenz von $S$. typhimurium DT104 (Tabelle 4, 5), dessen wichtigstes Merkmal eine breite Antibiotika-Multiresistenz von $76,0 \%$ bzw. $87,4 \%$ war, die vor allem die Antibiotika Tetracycline, Chloramphenicol, Streptomycin, Sulfonamid und die $\beta$-Lactam-Antibiotika der zweiten Generation betrifft (Tabelle 6). In geringem Maße wurde eine zusätzliche Resistenz gegen Trimethoprim (o,5\%/2,3\%) festgestellt.

Wie aus den Tabellen 4 und 5 zu entnehmen ist, spielt bei den übrigen Salmonellen vor allem die Resistenz gegen
Tetracycline $(19,4 \% / 23,3 \%)$ und Sulfonamid $(81,4 \% / 79,0 \%)$ bei einem Anteil multiresistenter Stämme von 19,0\% bzw. $21,5 \%$ eine Rolle. Bezogen auf alle Salmonellen ergab sich ein ähnlicher Anteil multiresistenter Stämme. Wichtig ist, daß bisher bei Salmonellen nur vereinzelt Resistenzen gegen Ciprofloxacin $(0,1 \% / 0,2 \%)$ und Cefotaxim (o,1\%/ $0,1 \%)$ beobachtet wurden.

\section{Diskussion}

Aufgrund der nach wie vor großen gesundheitspolitischen Bedeutung der Salmonellose in Deutschland ist deren epidemiologische Überwachung sowohl als Basis für Prävention und Bekämpfung wie auch als Modell der durch Lebensmittel übertragenen Infektionen zwingend erforderlich. Geeignete Methoden der epidemiologischen Überwachung sind dabei die Meldungen nach BSeuchG, die Nutzung anderer Datenquellen, wie z.B. die Auswertungen der
Untersuchung von Salmonella-Stämmen, die dem NRZ aus allen Regionen Deutschlands zur Typisierung zugeschickt werden sowie andere gezielte infektionsepidemiologische Studien. Besondere Bedeutung dabei nehmen die Sentinel-Studien ein, die unter definierten Bedingungen durchgeführt werden und deshalb ausgehend von einer Stichprobe Rückschlüsse auf die Gesamtheit erlauben.

Da die Einsendungen an das NRZ nur teilweise nach systematischen und/oder epidemiologischen Gesichtspunkten, sondern eher mehr zufällig und aus diagnostischen Gründen erfolgen, repräsentieren sie per se nicht die wahren epidemiologischen Verhältnisse in Deutschland. Zur fundierten Einschätzung der Salmonellose in Deutschland wurden deshalb die Daten des NRZ mit denen aus einer Sentinel-Studie über Vorkommen, Verbreitung und Typenspektrum von Salmonellen im Einzugsgebiet eines privat niedergelassenen Laboratoriums verglichen. Außerdem erfolgte zusätzlich ein weiterer Vergleich mit den Meldungen nach BSeuchG auf der Grundlage der Meldungen aus den neuen Bundesländern und Berlin.

Zur Einschätzung der Salmonellose in Deutschland 1997 wurden im Rahmen der Sentinel-Studie 1608 SalmonellaStämme isoliert und typisiert. Diese Ergebnisse wurden mit den 4321 dem NRZ zugeschickten humanen SalmonellaIsolaten und den 29326 nach BSeuchG aus den neuen Bundesländern und Berlin gemeldeten Salmonellen verglichen. Beurteilt wurden im einzelnen die monatliche Verteilung und Inzidenz der Salmonellose, die altersspezifische Morbidität der Salmonellose, Vorkommen und Häufigkeit der Salmonella-Serovare, Vorkommen und Häufigkeit der Lysotypen von S. enteritidis und S. typhimurium, Erfassung von Inzidenzsteigerungen und Ausbrüchen, Antibiotikaresistenz der Salmonellen.

Aus den vergleichenden Analysen der Sentinel-Studie, den Einsendungen an das NRZ und den Meldungen nach BSeuchG ergaben sich als wichtigste Resultate: 
Tabelle 3

Vorkommen und Häufigkeit der Lysotypen von S. typhimurium

Vergleich der Sentinel-Studie (S-St) und Typisierung humaner Isolate im NRZ 1997

\begin{tabular}{lrr} 
& S-St & NRZ \\
\hline Gesamtzahl der typisierten S. typhimurium & 635 & 1793 \\
Gesamtzahl der Lysotypen & 30 & 41 \\
Zahl der häufigsten Lysotypen ( $\mathrm{n} \geq 1 \%)$ & 13 & 15 \\
\hline
\end{tabular}

$\begin{array}{llll}\text { Lysotyp } & \text { S-St } & \text { Anteil in \% } & \text { Inzidenz* (pro } 100000 \mathrm{EW} \text { ) }\end{array}$

\begin{tabular}{lccr}
\hline DT 104 & $31,5^{1}$ & 38,1 & 20,0 \\
DT 120 & 8,8 & 10,4 & 5,6 \\
DT 012 & 8,3 & 5,9 & 5,3 \\
DT 193 & 8,2 & 9,6 & 5,2 \\
DT 170 & $9,6^{2}$ & 6,2 & 6,1 \\
DT 017 & 4,7 & 3,0 & 3,0 \\
ut DT-PTU 302 & 2,8 & 5,2 & 1,8 \\
DT 186 & $10,7^{3}$ & 1,1 & 6,8 \\
DT 15 a & 1,9 & 0,9 & 1,2 \\
DT 177 & 1,4 & 1,1 & 0,9 \\
DT 066 & 1,1 & 1,0 & 0,7 \\
RDNC & 0,9 & 1,1 & 0,6 \\
übrige & 5,8 & 11,7 & 3,7 \\
\hline Total & 100,0 & 100,0 & 63,5 \\
\hline
\end{tabular}

*aus den Daten der Sentinel-Studie berechnet

${ }^{1}$ beinhaltet einen Ausbruch mit 26 Fällen

2 beinhaltet einen Ausbruch mit 12 Fällen

3 beinhaltet einen Ausbruch mit 56 Fällen
4. Im Gegensatz dazu stimmten die Inzidenzraten der Salmonellose mit 160,8/165,4 sowie die Inzidenzraten der Salmonella-Infektionen durch die häufigsten Serovare in der SentinelStudie und den Meldedaten sehr gut überein (Abb. 4). Beachtenswert ist, daß die für das gesamte Bundesgebiet ermittelte Inzidenzrate der Salmonellose lediglich bei 128,4 lag [11]. Dies spricht entweder für eine mangelnde Meldedisziplin oder für eine unterschiedliche Salmonella-Morbidität in den verschiedenen Landesteilen. Die Unterschiede zwischen Sentinel-Studie, Typisierergebnissen des NRZ und Meldung nach BSeuchG bezüglich der monatlichen Verteilung der Salmonellose (Abb. 1) und der monatlichen Inzidenz der Salmonellose (Abb. 2) im September und Oktober ergeben sich aus den unvermeidbaren Verzögerungen der Datenerfassung bei den Typisierergebnissen und den Meldungen gegenüber der aktuellen Erfassung im Rahmen der Sentinel-Studie.

Insgesamt unterstützt zwar gute Übereinstimmung die Plausibilität, sagt aber nichts über die Validität der Datenquellen selbst aus. Auch für die Daten des Sentinel-Labors ist nicht bewertbar, inwieweit sie die „reale Situation im Einzugsgebiet" repräsentieren oder welches „Underreporting“ auch dort vorliegt.

1. Die simultane Auswertung der Sentinel-Studie, der NRZ-Daten und der Meldungen nach BSeuchG ermöglichte eine verbesserte Einschätzung der Salmonellose in Deutschland. Da die Bewertung auf der Basis von drei Datenquellen erfolgte, waren Aussagen mit größerer Sicherheit möglich.

2. Sentinel-Studie, NRZ- und Meldedaten validieren sich in bestimmten Analyse- und Aussagebereichen wechselseitig (z.B. saisonaler Verlauf, Serovar-Struktur). Darüber hinaus lieferten die Sentinel-Studie, wie auch die NRZ-Daten, Informationen über die Verteilung der Lysotypen sowie der Antibiotikaresistenz. Vergleicht man die Ergebnisse der Sentinel-Studie mit denen der Typisierungen im NRZ und denen der Meldungen, so ergibt sich eine erstaunliche Homogenität und Vergleichbarkeit der erzielten Daten. Allerdings sollten sowohl Überein- stimmungen als auch Abweichungen zwischen den Raten und Strukturen der verglichenen Datenquellen sehr vorsichtig bewertet werden.

3. Einschränkungen in der Aussage ergaben sich in der Sentinel-Studie sowohl aufgrund der begrenzten, in die Untersuchung einbezogenen Population als auch aufgrund teilweise erheblicher regionaler Unterschiede bei den nachgewiesenen Serovaren und aufgrund des eingeschränkten Zeitraums der Analyse. So erfaßte die Sentinel-Studie mit 40 Serovaren nur ein schmaleres Spektrum, und S. typhi sowie S. paratyphi $B$ wurden nicht gefunden. Aus den Einsendungen an das NRZ wurden dagegen 105 Serovare bestimmt, die Meldungen registrierten 141 Serovare. Ein wesentlicher Vorteil der Sentinel-Studie gegenüber anderen Datenquellen ist die sofortige Erkennung von Inzidenzsteigerungen und Ausbrüchen.
5. Obwohl durch Salmonellen bedingte Ausbrüche gegenwärtig relativ häufig sind und sich in ihrem Ablauf weitgehend gleichen, sind die hier beschriebenen Inzidenzsteigerungen durch einige Besonderheiten gekennzeichnet. Die Erkrankungshäufungen durch bestimmte S. typhimurium-Klone wurden nur als Ausbruch in zwei benachbarten Kreisen erkannt, weil durch die gerade in dieser Region laufende Sentinel-Studie alle isolierten S. typhimurium-Stämme dem NRZ zur Lysotypie übergeben wurden. Die daraufhin von den zuständigen Behörden veranlaßten intensiven Lebensmitteluntersuchungen belegten durch den Nachweis des gleichen Klons im Schweinehackfleisch den Zusammenhang mit den Erkrankungen. Anzustreben wären Abläufe, die weniger zufallsabhän- 
Tabelle 4

Antibiotikaresistenz der Salmonellen

Vergleich Sentinel-Studie (S-St) 1997 und Typisierung humaner Isolate im NRZ 1997

\begin{tabular}{|c|c|c|c|c|c|c|c|c|c|c|c|c|}
\hline \multirow[t]{2}{*}{ Erreger } & \multirow{2}{*}{$\begin{array}{l}\text { Vergleich } \\
\text { Studie }\end{array}$} & \multirow[t]{2}{*}{ getestete Stämme } & \multicolumn{10}{|c|}{ Antibiotikaresistenz in \% } \\
\hline & & & AMP & SXT & СTX & CIP & OTE & CMP & SMZ & STR & GEN & andere \\
\hline \multirow[t]{2}{*}{ S. enteritidis } & S-St & 715 & 1,1 & 0,3 & 0,1 & 0,0 & 0,7 & 0,6 & 74,5 & 0,3 & 0,0 & 1,5 \\
\hline & NRZ & 1637 & 2,0 & 0,8 & 0,1 & 0,0 & 2,2 & 0,7 & 71,8 & 1,6 & 0,4 & 2,6 \\
\hline \multirow[t]{2}{*}{ S.typhimurium } & S-St & 635 & 32,3 & 3,8 & 0,2 & 0,3 & 43,5 & 29,1 & 74,2 & 41,7 & 0,2 & 32,9 \\
\hline & NRZ & 1793 & 48,2 & 7,6 & 0,2 & 0,1 & 59,9 & 38,6 & 77,4 & 57,1 & 1,8 & 48,5 \\
\hline \multirow[t]{2}{*}{ S.typhimurium DT104 } & S-St & 200 & 73,5 & 2,0 & 0,0 & 0,0 & 74,5 & 71,5 & 97,5 & 93,5 & 0,0 & 74,0 \\
\hline & NRZ & 683 & 85,4 & 3,2 & 0,1 & 0,1 & 86,8 & 83,7 & 98,1 & 96,3 & 0,1 & 85,4 \\
\hline \multirow[t]{2}{*}{ S.typhimurium non DT104 } & S-St & 435 & 13,3 & 4,6 & 0,2 & 0,5 & 29,2 & 9,7 & 63,4 & 17,9 & 0,2 & 14,0 \\
\hline & NRZ & 1110 & 25,3 & 10,4 & 0,2 & 0,1 & 43,3 & 10,8 & 64,6 & 32,9 & 2,9 & 25,8 \\
\hline \multirow[t]{2}{*}{ S.typhi } & S-St & 0 & 0,0 & 0,0 & 0,0 & 0,0 & 0,0 & 0,0 & 0,0 & 0,0 & 0,0 & 0,0 \\
\hline & NRZ & 34 & 8,8 & 8,8 & 0,0 & 0,0 & 11,8 & 11,8 & 32,4 & 8,8 & 0,0 & 8,8 \\
\hline \multirow[t]{2}{*}{ übrige Salmonellen } & S-St & 258 & 5,4 & 10,1 & 0,0 & 0,0 & 19,4 & 12,8 & 81,4 & 19,0 & 0,8 & 9,7 \\
\hline & NRZ & 857 & 9,8 & 10,3 & 0,0 & 0,8 & 23,3 & 10,6 & 79,0 & 20,0 & 2,3 & 14,7 \\
\hline \multirow[t]{2}{*}{ alle SalmonellenS-St } & 1608 & 14,1 & 3,2 & 0,1 & 0,1 & 20,6 & 13,8 & 75,5 & 19,7 & 0,2 & 15,2 & \\
\hline & NRZ & 4321 & 22,8 & 5,6 & 0,1 & 0,2 & 30,4 & 18,5 & 75,2 & 28,3 & 1,4 & 24,1 \\
\hline
\end{tabular}

Symbole der Antibiotikaresistenz: $A M P=$ Ampicillin, SXT=Trimethoprim/Sulfamerazin (1:20), CTX=Cefotaxim, CIP=Ciprofloxacin, OTE=0xytetracyclin, $C M P=C h l o r-$ amphenicol, $S M Z=$ Sulfamerazin, $S T R=S$ treptomycin, GEN=Gentamicin

Andere: $A M K=$ Amikazin, $C A Z=C$ eftazidim, COX=Cefoxitin, $C T M=$ Cefotiam, $K A N=$ Kanamycin, MEZ=Mezlocillin, MUS=Mezlocillin/Sulbactam, NOT=Nourseothricin, PTB=Piperacillin/Tazobactam

gig sind. Eine gut funktionierende Surveillance sollte derartige Erkrankungsgeschehen auch bei Streuung der Einzelerkrankungen über zwei Landkreise rechtzeitig erkennen, was nur durch die Anwendung der epidemiologischen Laboratoriumsmethoden an einer genügenden Anzahl von ausgewählten Erregerisolaten möglich ist. Zu beachten bleibt, daß das Erfassen eines Ausbruchs und das Klären der Ursache als Grundlage wirksamer antiepidemischer und präventiver Maßnahmen nur in enger Zusammenarbeit zwischen behandelnden Ärzten, Mikrobiologen und Epidemiologen möglich ist.

\section{„Prognostische Einschätzungen der vergangenen Jahre, daß der führende Epidemiestamm S. enteriti- dis LT 4/6 rückläufig ist, haben sich 1997 nicht erfüllt."}

6.S. enteritidis-Stämme spielen nach wie vor epidemiologisch die wichtigste Rolle in Deutschland und Europa. Prognostische Einschätzungen der vergangenen Jahre, daß der führende Epidemiestamm S. enteritidis LT 4/6 rückläufig ist und durch einen neuen aufkommenden Epidemiestamm $S$. typhimurium DT104 ersetzt wird, haben sich 1997 nicht erfüllt [12]. Der $S$. enteridis-Epidemiestamm LT $4 / 6$ hat nach wie vor in allen europäischen Ländern die höchste Inzidenz der Salmonellen. Die Erkrankungen werden vorwiegend durch den Verzehr kontaminierter Eier und Eiprodukte sowie Geflügel ausgelöst. Die weiterhin hohe Inzidenz von S. enteritidis LT $4 / 6$ weist nicht nur auf einen hohen Durchseuchungsgrad mit $S$. enteritidis hin, sondern unterstreicht auch die Wiederzunahme hygienischer Küchenfehler als Ursache von Ausbrüchen. Offenbar ist die in den Jahren 1992-1994 erreichte Aufmerksamkeit der Öffentlichkeit wieder verlorengegangen.

Der neue Epidemiestamm $S$. typhimurium DT104 breitete sich nach den Ergebnissen des NRZ 1997 nur unwesentlich aus [15]. Der nach den Einsendungen an das NRZ ermittelte hohe Anteil von $38,1 \%$ der typisierten S. typhimurium-Stämme (Abb. 3) ist sicher wesentlich auf die Bitte des NRZ zurückzuführen, multiresistente S. typhimurium-Isolate zur Typisierung einzuschicken [13]. Als Infektionsquelle waren anfangs ausschließlich Rinder, in letzter Zeit zunehmend Schweine und andere Tierarten betroffen [14].

7. Im Gegensatz zu den Shigellen sind die Antibiotikaresistenzverhältnisse bei Salmonellen, insbesondere bei $S$. enteritidis, sehr gering (Tabelle 4, 5). Die im NRZ beobachtete Zunahme der Antibiotikaresistenz bei Salmonellen in den Jahren 1996/97 war fast ausschließlich auf die erhöhte Inzidenz von S. typhimurium DT104 zurückzuführen. Dieser SalmonellaDT104-Stamm zeigt eine multiple Antibiotikaresistenz (Tabelle 5, 6) und trägt seit dem Jahr 1990 wesentlich zum Phänomen einer rapiden Ausbreitung der Antibiotikaresistenz bei Salmonellen bei. Neben seiner breiten Palette der Antibiotikaresistenz zeigt dieser Epidemiestamm Variationen im Plasmid- und Resistenzprofil, was jedoch bisher nicht zur Bildung bestimmter Subklone geführt hat [15]. 
Originalien und Übersichtsarbeiten

Tabelle 5

Sensibilität sowie Ein- und Mehrfachresistenz der Salmonellen

Vergleich Sentinel-Studie (S-St) 1997 und Typisierung humaner Isolate im NRZ 1997

\begin{tabular}{|c|c|c|c|c|c|c|}
\hline \multirow[t]{2}{*}{ Erreger } & \multirow{2}{*}{$\begin{array}{l}\text { Vergleich } \\
\text { (Studie) }\end{array}$} & \multirow[t]{2}{*}{ getestete Stämme } & \multirow[b]{2}{*}{ sensible } & \multicolumn{3}{|c|}{ Sensible und resistente Stämme in \% } \\
\hline & & & & $\begin{array}{l}\text { einfach- } \\
\text { resistent }\end{array}$ & $\begin{array}{l}\text { zweifach- } \\
\text { resistent }\end{array}$ & $\begin{array}{l}\text { multi- } \\
\text { resistent }\end{array}$ \\
\hline \multirow[t]{2}{*}{ S. enteritidis } & S-St & 715 & 24,9 & 73,1 & 0,7 & 1,3 \\
\hline & NRZ & 1637 & 27,1 & 68,1 & 2,0 & 2,8 \\
\hline \multirow[t]{2}{*}{ S.typhimurium } & S-St & 635 & 16,9 & 30,7 & 15,9 & 36,5 \\
\hline & NRZ & 1793 & 16,2 & 19,2 & 10,0 & 54,6 \\
\hline \multirow[t]{2}{*}{ S. typhimurium DT104 } & S-St & 200 & 0,5 & 2,5 & 21,0 & 76,0 \\
\hline & NRZ & 683 & 1,2 & 1,6 & 9,8 & 87,4 \\
\hline \multirow[t]{2}{*}{ S.typhimurium non DT104 } & S-St & 435 & 24,4 & 43,7 & 13,6 & 18,4 \\
\hline & NRZ & 1110 & 25,4 & 30,1 & 10,1 & 34,4 \\
\hline \multirow[t]{2}{*}{ S.typhi } & S-St & 0 & 0,0 & 0,0 & 0,0 & 0,0 \\
\hline & NRZ & 34 & 67,6 & 20,6 & 0,0 & 11,8 \\
\hline \multirow[t]{2}{*}{ übrige Salmonellen } & S-St & 258 & 16,7 & 48,8 & 16,7 & 19,0 \\
\hline & NRZ & 857 & 20,3 & 52,6 & 13,0 & 21,5 \\
\hline \multirow[t]{2}{*}{ alle Salmonellen } & S-St & 1608 & 20,4 & 52,5 & 9,3 & 18,0 \\
\hline & NRZ & 4321 & 21,5 & 44,4 & 7,5 & 28,1 \\
\hline
\end{tabular}

Demzufolge ist hinsichtlich der Antibiotikaresistenzentwicklung der Salmonellen eine andere epidemiologische Beurteilung erforderlich, als wenn sich in Deutschland verschiedene mehrfachresistente Salmonella-Klone ausbreiten würden. Die multiple Antibiotikaresistenz von $S$. typhimurium DT104 ist im Gegensatz $\mathrm{zu}$ den Shigellen und auch anderen Salmonellen nicht durch Plasmide, sondern chromosomal bedingt. Wichtig ist, daß bisher im Gegensatz zu Großbritannien [10] in Deutschland die Ciprofloxacin-Resistenz sowohl von S. typhimurium DT104 als auch von anderen Salmonellen keine Rolle spielt (Tabelle 4).

8. Laborgestützte infektionsepidemiologische Methoden sind nicht von anderen epidemiologischen Untersuchungen zu trennen, bedürfen jedoch stets der engen Zusammenarbeit mit der Primärdiagnostik und den epidemiologisch vermittelnden Gesundheitsämtern. Diese Grundstruktur eines noch zu schaffenden epidemiologischen Netzwerkes soll im Rahmen der
Fortführung der Sentinel-Studie im Jahr 1998 zur weiteren Qualifikation der epidemiologischen Daten über die Salmonellose in Deutschland, der Erfassung der Populationsstruktur, der Antibiotikaresistenz und der Aufklärung der nationalen und internationalen Infektionswege und -quellen erprobt, intensiviert und erheblich ausgebaut werden.

Tabelle 6

Dominierende Resistenzmuster von S. typhimurium DT104

Vergleich Sentinel-Studie (S-St) 1997 und Typisierung humaner Isolate im NRZ 1997 sowie England und Wales (E) 1996

\begin{tabular}{|c|c|c|c|c|c|c|c|c|c|}
\hline \multirow[t]{2}{*}{ Vergleich } & \multirow[t]{2}{*}{ Jahr } & \multirow[t]{2}{*}{ getestete Stämme } & \multicolumn{2}{|c|}{ Resistenz } & \multirow[b]{2}{*}{ ACSSuT } & \multirow[b]{2}{*}{ ACSSuTTm } & \multicolumn{2}{|c|}{ Resistenzmuster in \% } & \multirow[b]{2}{*}{ andere } \\
\hline & & & $\mathbf{n}$ & $\%$ & & & ACSSuTCp & ACSSuTTmCp & \\
\hline S-St & 1997 & 200 & 99 & 99,5 & 68,0 & 0,5 & 0,0 & 0,0 & 31,0 \\
\hline NRZ & 1997 & 683 & 675 & 98,8 & 79,9 & 2,3 & 0,0 & 0,0 & 16,5 \\
\hline$E^{*}$ & 1996 & 4006 & 3935 & 98,2 & 58,0 & 21,0 & 13,0 & 1,0 & 6,0 \\
\hline
\end{tabular}

*Threlfall et al. [10]

Symbole der Antibiotikaresistenz: A=Ampicillin, C=Chloramphenicol, S=Streptomycin, Su=Sulfonamide, $T=$ Tetracycline, Tm=Trimethoprim/Sulfamerazin, C $p=$ Ciprofloxacin 


\section{Literatur}

1. Deutsche Gesellschaft für Hygiene und Mikrobiologie (Hrsg) (1991) Verfahrensrichtlinien für die mikrobiologische Diagnostik. Fischer, Stuttgart, New York

2. Dusch H (1996) Salmonellen. In:Laboratoriumsmedizin 1996; 20 Diagnostische Bibliothek Nr. 44, S 1-12

3. Klare I, Witte W (1988) Mikrobouillonverdünnungstest zur standardisierten Bestimmung minimaler Hemm- und minimaler bakterizider Konzentrationen antibakterieller Chemotherapeutika bei schnellwachsenden aeroben Bakterien. Z Gesamte Hyg 34:111-115

4. Anderson ES, Williams REO (1956) Bacteriophage typing of enteric pathogens and staphylococci and its use in epidemiology. J Clin Path 9:94-127

5. Anderson ES, Ward LR, de Saxe JDH (1977) Bacteriophage-typing designation of Salmonella typhimurium.J Hyg 78:297

6. Ward LR, de Saxe JDH, Rowe B (1987) A phage-typing schema for Salmonella enteritidis. Epidem Inf 99: 291-294

7. Laszlo VG, Csorian ES, Milch H (1985) Phage types and epidemiological significance of Salmonella enteritidis strains in Hungary between 1976 and 1983. Acta Microbiol Hung 32:321-340

8. Heinemann L, Sinnecker H (Hrsg) (1994) Epidemiologische Arbeitsmethoden. Fischer, Jena

9. Robert Koch-Institut Zwei SalmonelloseAusbrüche durch S. typhimurium in zwei benachbarten Landkreisen Thüringens. Epidemiologisches Bulletin 24/97,S 161-162
10. Threlfall EJ, Ward LR, Rowe B (1997) Increasing incidence of resistance to trimethoprim and ciprofloxacin in epidemic Salmonella typhimurium DT104 in England and Wales. Surveillance 2:81-84

11. Robert Koch-Institut (1998) Zur Situation bei ausgewählten meldepflichtigen Infektionskrankheiten im Jahr 1997. Teil 1: Gastroenteritiden (I)-Salmonellose. Epidemiologisches Bulletin 8/98: 5 47-48

12. Tschäpe $H$, Gericke $B$, Prager $R$, Liesegang $A$ (1997) The up and down of Salmonella enteritidis in Germany. In:Saeed AM (ed) Purdue Univ Press

13. Robert Koch-Institut (1996) Verbreitung von Salmonella typhimurium DT104 jetzt auch in Deutschland. Epidemiologisches Bulletin 24/96: 165

14. Liesegang A, Prager R, Streckel W, Rabsch W, Gericke B, Seltmann G, Helmuth R, Tschäpe H (1996) Wird der Salmonella-entericaStamm DT104 des Serovars Typhimurium der neue führende Epidemietyp in Deutschland? Robert Koch-Institut, InfFo I/97: 6-10

15. Prager $R$, Liesegang $A$, Rabsch $W$, Gericke $B$, Helmuth R, Thiel W, Ward L, Tschäpe H (1998) Clonal relationship of Salmonella enterica, serovar Typhimurium, phage type DT104 and its epidemiological implication. J Med Microbiol (im Druck)

\section{Pschyrembel - Therapeutisches Wörterbuch}

824S., 482 Abb., 207 Tab. Berlin, New York:Walter de Gruyter Verlag.DM 78,-

Nahezu jeder medizinisch Tätige in Deutschland kennt den „Pschyrembel", das klinische Wörterbuch, als unentbehrliches Nachschlagewerk. Derselben Idee und Philosophie folgend gibt es nun noch einen „Pschyrembel ", nämlich ein Therapeutisches Wörterbuch.

Von einer bekannten Diagnose (i.e. Krankheit, Befindlichkeitsstörung, Symptom, Syndrom) in alphabetischer Anordnung ausgehend, werden die therapeutischen Möglichkeiten erläutert.Diskutiert werden Behandlungsindikationen, pharmakologische, operative und sonstige,z.B. physikalische Therapiemaßnahmen sowie therapeutische Stufenpläne. Hingewiesen wird auf aktuelle Neuentwicklungen im jeweiligen Bereich und auf Selbsthilfegruppen (mit Adressen). Tabellen, Schaubilder und grafische Darstellungen werden zur Veranschaulichung und besseren Übersicht eingesetzt. Das 824 Seiten starke Nachschlagewerk enthält rund 700 Diagnosen und 300 therapeutische Verfahren. Eine stichprobenartige Überprüfung der Aktualität verläuft ohne wesentliche Beanstandungen, die umfangreiche Liste der beitragenden Autoren ist durchaus vertrauenserweckend.

Das Therapeutische Wörterbuch ersetzt natürlich nicht die praktische Erfahrung und die kontinuierliche Weiterbildung und sollte daher sicher nicht von Unerfahrenen als Rezeptbuch zur Behandlung/Weiterbildung/Selbstbehandlung mißbraucht werden. Es gewährt jedoch einen breiten Überblick über die aktuellen therapeutischen Möglichkeiten und Strategien (bei bekannter Diagnose!!) und liefert zusätzliche hilfreiche Tips und Informationen. Für therapeutisch, pflegend und beratend im Bereich der Krankenversorgung Tätige, aber auch z.B. für Mitarbeiter von Krankenkassen und gutachterliche Tätigkeiten ist der neue „Therapie“-Pschyrembel wahrscheinlich eine sinnvolle und lohnende Investition (eine CD-ROM-Version ist in Vorbereitung). 